\title{
DISSIPAÇÃO DO INSETICIDA TIAMETOXAM EM SOLOS DA REGIÃO DE LAVRAS-MG
}

\author{
ANA PAULA FONSECA MAIA DE URZEDO* \\ RENÊ LUÍS DE OLIVEIRA RIGITANO** \\ MÁRIO CÉSAR GUERREIRO*** \\ NÉLIO RICARDO DO AMARAL CASTRO****
}

\begin{abstract}
O objetivo deste trabalho foi investigar a dissipação do tiametoxam em amostras de Latossolos do município de Lavras, MG (Brasil), bem como verificar a influência da calagem, fosfatagem, atividade microbiana e umidade do solo nesse processo. Subamostras dos solos foram incubadas com o inseticida a $25^{\circ} \mathrm{C}$, sendo periodicamente analisadas com relação à quantidade remanescente de tiametoxam (por cromatografia a líquido). A dissipação do tiametoxam nos solos estudados seguiu cinética de primeira ordem (valores de meiavida entre 117 e 301 dias), revelando alta estabilidade do composto. A calagem do solo diminuiu a taxa de dissipação do inseticida no caso do Latossolo Vermelho Amarelo distrófico, sendo esse efeito parcialmente antagonizado pela fosfatagem. No caso do Latossolo Vermelho distrófico, o efeito da calagem não foi significativo. A taxa de dissipação do tiametoxam diminuiu sensivelmente em subamostras de Latossolo previamente submetidas à autoclavagem, mostrando que a atividade microbiana desempenha importante papel na degradação do composto.
\end{abstract}

PALAVRAS-CHAVE: TIAMETOXAM; DEGRADAÇÃO; LATOSSOLO.

* Química, Doutoranda em Química, Universidade Federal de Minas Gerais (UFMG), Belo Horizonte, MG (e-mail: anaurzedo@hotmail.com).

** Engenheiro Agrônomo, Ph. D., Professor Titular, Departamento de Entomologia, Universidade Federal de Lavras (UFLA), Lavras, MG (e-mail: rigitano@ufla.br).

*** Químico, D. Sc., Professor Adjunto, Departamento de Química, UFLA, Lavras, MG (e-mail: guerreiro@ufla.br).

**** Engenheiro Agrônomo, D. Sc. em Entomologia, UFLA, Lavras, MG (e-mail: nelio_castro@yahoo.com.br). 


\section{INTRODUÇÃO}

A aplicação de inseticidas no solo constitui prática muito comum na agricultura brasileira, especialmente na cafeicultura. Depois de aplicados no solo, os inseticidas ficam sujeitos aos processos de adsorção nos colóides do solo, absorção pelas raízes, degradação, lixiviação, etc. A lixiviação dos compostos pode resultar no transporte de seus resíduos até minas ou poços d'água, como constatado nos Estados Unidos da América (DOMAGALSKY e DUBROSKY, 1992), na Europa (LEISTRA e BOESTEN, 1989) e na região de Maria da Fé em Minas Gerais (RIGITANO e GOUVÊA, 1995).

Quanto menor for a adsorção do pesticida nos colóides de determinado solo e menor for a sua taxa de degradação nesse solo, maior será sua lixiviação potencial. Vários fatores afetam a taxa de degradação dos pesticidas em solos, dentre os quais a natureza química do composto e parâmetros associados ao solo, como temperatura, umidade, pH, atividade microbiana, etc. (DÍAZ et al., 1995; RACKE et al., 1997; BROMILOW, EVAN e NICHOLLS, 1999). O termo dissipação tem sido preferivelmente empregado para expressar a diminuição da quantidade do pesticida no solo em função do tempo. Os métodos utilizados na determinação da quantidade remanescente dos compostos, na maioria dos casos, não distinguem degradação propriamente dita e volatilização, formação de resíduo ligado, etc. (LAVORENTI, PRATA e REGITANO, 2003).

O inseticida tiametoxam, composto da classe dos neonicotinóides, foi introduzido no Brasil em 1999 para uso em várias culturas. Por apresentar propriedades sistêmicas (MAIENFISCH et al., 2001), o tiametoxam não é utilizado apenas na forma de pulverização. Sua aplicação no solo visa o controle de insetos-praga da parte aérea das plantas, como é o caso do bicho-mineiro em folhas de cafeeiro. $O$ uso desse inseticida nas regiões cafeeiras vem aumentando rapidamente, embora a sua dissipação em solos brasileiros não tenha sido relatada.

Estudos sobre a dissipação do tiametoxam em solos são escassos e restritos a solos norteamericanos e europeus. ANTUNES-KENYON e KENNEDY (2001) relataram que sob condições de fotodegradação, a estabilidade do tiametoxam em solos é moderada com valores de meia-vida em torno de 50 dias. Já em condições aeróbicas e na ausência de luz, os valores de meia-vida alcançaram 385 dias. Segundo esses autores, as propriedades e características do tiametoxam o enquadram como contaminante potencial da água do subsolo. Tal assertiva foi baseada na baixa adsorção do composto nos colóides do solo e na sua alta estabilidade nos solos estudados. ROBINSON (2001) relatou valores de meia-vida do tiametoxam superiores a 180 dias em solo arenoso dos Estados Unidos. MAIENFISCH et al. (2001), pesquisadores da empresa fabricante do tiametoxam, relataram que em condições de laboratório a meia-vida do tiametoxam em solos varia de 34 a 75 dias, os quais podem ser aumentados em três vezes sob condições desfavoráveis à degradação.

O presente trabalho foi conduzido com o objetivo de investigar a dissipação do tiametoxam em solos brasileiros típicos, bem como verificar a influência da atividade microbiana, da calagem e/ou fosfatagem do solo nesse processo.

\section{MATERIAL E MÉTODOS}

\subsection{SOLOS UTILIZADOS}

Foram coletadas amostras de solos das classes Latossolo Vermelho Amarelo Distroférrico $\left(\operatorname{LVA}_{\mathrm{df}}\right)$, Latossolo Vermelho Amarelo Distrófico $\left(\operatorname{LVA}_{\mathrm{d}}\right)$ e Latossolo Vermelho Distrófico $\left(\mathrm{LV}_{\mathrm{d}}\right)$, sob vegetação natural, localizados na região de Lavras, MG. Amostras desses solos, correspondentes à profundidade de 0 a $20 \mathrm{~cm}$, foram secas ao ar, destorroadas e peneiradas em malha de $2 \mathrm{~mm}$. 
Tendo conhecimento do grau de umidade das amostras, determinado em estufa a $105^{\circ} \mathrm{C}$ durante 24 horas, acondicionou-se quantidade correspondente a $50 \mathrm{~g}$ de solo seco em copos de vidro do tipo americano. De cada solo foram preparadas 12 subamostras, correspondentes a 2 repetições e 6 datas de amostragem $(0,30,60,90,120$ e 210 dias após a aplicação do composto).

As subamostras dos solos $\mathrm{LVA}_{\mathrm{df}}, \mathrm{LVA}_{\mathrm{d}}, \mathrm{LV}_{\mathrm{d}}$ tiveram sua umidade ajustada a 19,4, 27,3 e 27,3\%, respectivamente, correspondentes à média entre o grau de umidade na capacidade de campo e no ponto de murcha permanente de cada solo (EMBRAPA, 1997). No caso do solo LVA df $_{\text {conduziu-se }}$ tratamento adicional, no qual o grau de umidade foi ajustado a $30 \%$, a fim de avaliar a influência do aumento da umidade do solo sobre a dissipação do tiametoxam.

As subamostras foram fortificadas com 0,25 mg de tiametoxam, mediante aplicação de $1 \mathrm{~mL}$ de solução aquosa preparada com padrão analítico do produto. O composto foi uniformizado nas amostras com auxílio de bastão de vidro e os copos tampados com papel alumínio perfurado para permitir a aeração. Os copos foram mantidos em câmaras climáticas a $25^{\circ} \mathrm{C}$, com fotoperíodo de 12 horas. A concentração do tiametoxam nas subamostras de solo $\left(5 \mathrm{mg} \mathrm{kg}^{-1}\right)$ foi estabelecida com base na dosagem do composto recomendada para o controle do bicho-mineiro do cafeeiro. As subamostras de solo tiveram sua umidade controlada pela adição de água a cada 2 ou 3 dias, em quantidade calculada mediante pesagem dos copos.

Para investigar a influência da atividade microbiana na dissipação do tiametoxam, doze subamostras do solo $\mathrm{LVA}_{\mathrm{df}}$ tiveram sua umidade acertada para 19,4\%. Posteriormente foram colocadas em frascos erlenmeyer, devidamente tampados. As amostras foram submetidas à autoclavagem a $120^{\circ} \mathrm{C}$ por dois períodos de 30 minutos, com intervalo de 8 horas entre os dois períodos, antes da aplicação do composto.

Os efeitos da calagem e/ou fosfatagem do solo sobre a dissipação do tiametoxam foram investigados em amostras do horizonte $A$ dos solos $L_{V A}$ e $L V_{d}$, previamente submetidas a esses tratamentos conforme NÓBREGA et al. (2005). Uma amostra de cada solo foi incubada com carbonato de cálcio, durante um mês, com umidade mantida em torno de $60 \%$ do volume total de poros. Determinouse a dose de $\mathrm{CaCO}_{3}$ aplicada mediante curva de incubação para estimativa da quantidade necessária para se elevar o pH a 6,5. Após esse período, metade da amostra que recebeu $\mathrm{CaCO}_{3}$ foi incubada por um mês com fosfato ácido de potássio, aplicado em concentrações correspondentes à capacidade máxima de adsorção de fósforo (550 e $1000 \mathrm{mg} \mathrm{kg}^{-1}$ para o $\mathrm{LVA}_{\mathrm{d}}$ e $\mathrm{LV}_{\mathrm{d}}$, respectivamente). Anteriormente à aplicação do tiametoxam procedeu-se a leitura do pH de subamostras dos solos, após agitação em solução de $\mathrm{CaCl}_{2}$ 0,01 M na proporção solo/solução de 1:2,5. A aplicação do inseticida e a condução desse experimento foram feitas conforme descrito anteriormente, mantendo-se o grau de umidade nas subamostras em $27,3 \%$.

Para a análise estatística dos resultados utilizou-se o programa Bio Estat (AYRES et al., 2000), efetuando-se o teste de homogeneidade de equações de regressão.

\subsection{PROCEDIMENTO ANALÍTICO}

Para a determinação do tiametoxam nas subamostras de solo adaptou-se metodologia desenvolvida pela Novartis Crop Protection AG (NOVARTIS, 1998). As subamostras de solo foram transferidas para frascos erlenmeyer de $500 \mathrm{~mL}$, aos quais adicionaram-se $150 \mathrm{~mL}$ de acetona p.a. Os frascos foram mantidos em mesa agitadora durante 1 hora e, em seguida, deixados em repouso por 2 horas para a decantação das partículas do solo. Transferiu-se alíquota de $1 \mathrm{~mL}$ do sobrenadante para balão de fundo redondo, acoplado a rotavapor, com banho a 50ㄷ para eliminação da mistura acetona e água. Os resíduos foram dissolvidos em $5 \mathrm{~mL}$ da mistura água:acetonitrila (65:35), filtrados em membrana PTFE $(0,45 \mu \mathrm{m})$. 
Efetuou-se a determinação quantitativa do tiametoxam em sistema de cromatografia a líquido de alta eficiência, marca Agilent - série 1100, operando com detector de ultravioleta ( $255 \mathrm{~nm}$ ). Utilizouse coluna LiChroCART ( $25 \mathrm{~cm}$ de comprimento e $3 \mathrm{~mm}$ de diâmetro interno), preenchida com LiChrospher RP-18, $5 \mu \mathrm{m}$. O fluxo da fase móvel $\left(\mathrm{H}_{2} \mathrm{O}\right.$ Milli-Q/acetonitrila-65/35) foi de $0,3 \mathrm{~mL} / \mathrm{min}$ e o volume de injeção de $20 \mu \mathrm{L}$. Nessas condições, o tempo de retenção do tiametoxam ficou em torno de 7 minutos. Obteve-se o padrão analítico do tiametoxam utilizado nas análises junto à Syngenta Proteção de Cultivos Ltda., tendo especificação de pureza acima de 99\%. Para avaliação da eficiência do método analítico foram realizados testes de recuperação do composto em amostras fortificadas nas concentrações de 0,5 e $5,0 \mathrm{mg} \mathrm{kg}^{-1}$.

\section{RESULTADOS E DISCUSSÃO}

Os testes de recuperação do tiametoxam revelaram eficiência média de $96 \pm 2 \%$, mostrando a adequabilidade do método analítico utilizado. Os resultados obtidos nas análises das subamostras de solo incubadas com o composto foram corrigidos de acordo com tal porcentagem de recuperação. As curvas de dissipação do tiametoxam nos solos estudados são apresentadas nas Figuras 1, 2 e 3.

\section{FIGURA 1 - CURVAS DE DISSIPAÇÃO DO TIAMETOXAM EM AMOSTRAS DE LATOSSOLO VERMELHO AMARELO DISTROFÉRRICO SUBMETIDAS A DIFERENTES TRATAMENTOS}

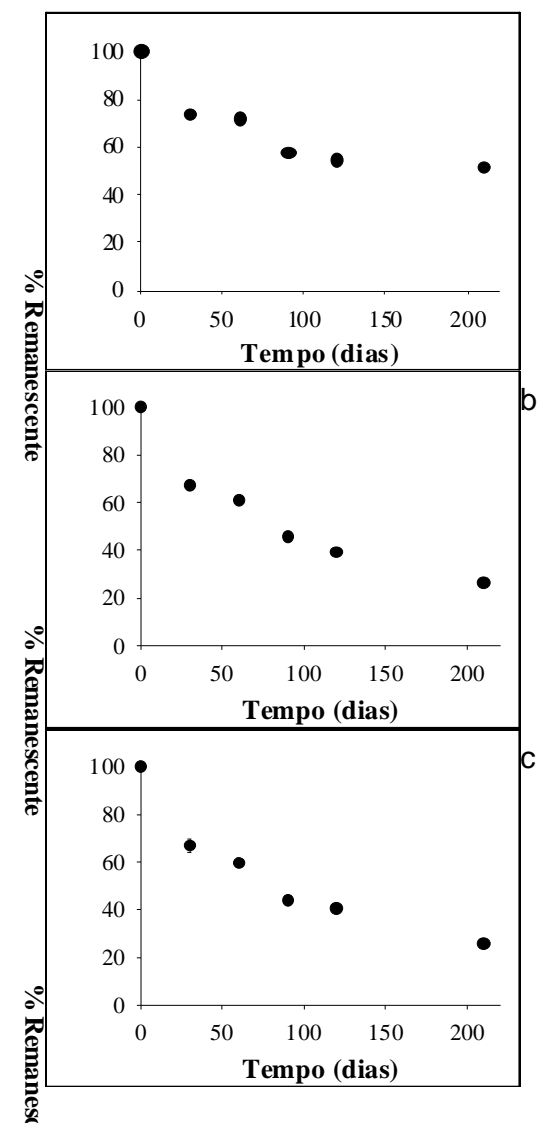

a) Solo esterilizado, com 19,4\% de umidade; b) Solo não-esterelizado, com 19,4\% de umidade; c) Solo nãoesterelizado, com $30 \%$ de umidade. 


\section{FIGURA 2 - CURVAS DE DISSIPAÇÃO DO TIAMETOXAM EM AMOSTRAS DE LATOSSOLO VERMELHO AMARELO DISTRÓFICO SUBMETIDAS A DIFERENTES TRATAMENTOS}

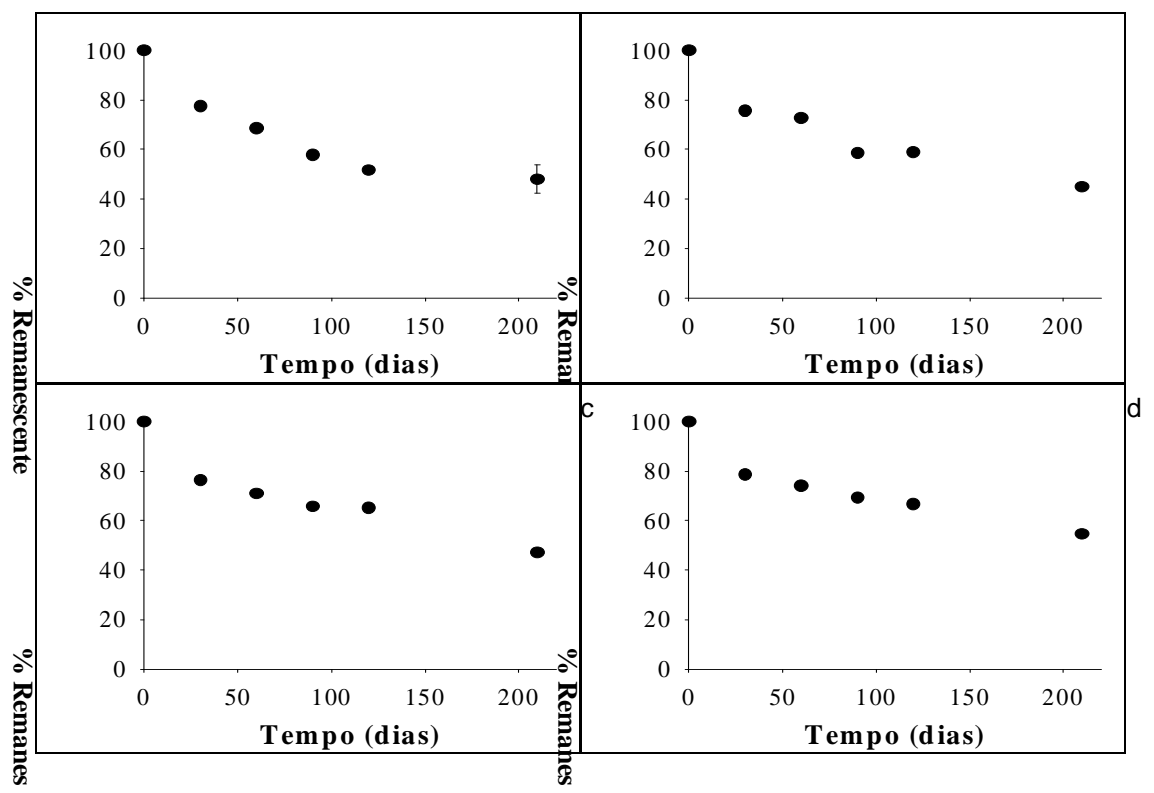

a) Sem calagem e fosfatagem; b) Sem calagem e com fosfatagem; c) com calagem e fosfatagem; d) Com calagem e sem fostagem.

\section{FIGURA 3 - CURVAS DE DISSIPAÇÃO DO TIAMETOXAM EM AMOSTRAS DE LATOSSOLO VERMELHO DISTRÓFICO SUBMETIDAS À CALAGEM (a) OU NÃO (b)}

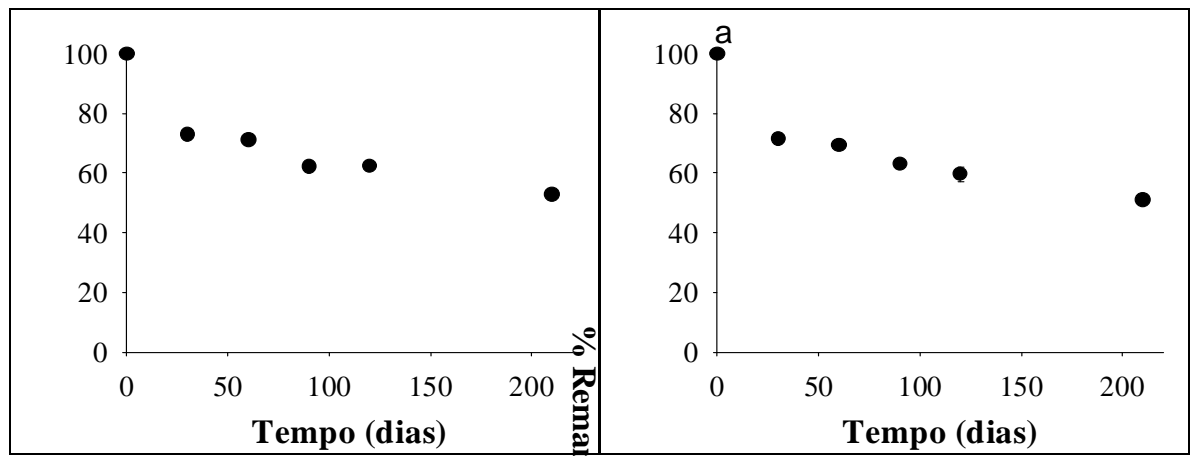

b

Observou-se que a dissipação do tiametoxam nos diferentes solos/tratamentos seguiu cinética de primeira ordem, uma vez qua a quantidade remanescente do inceticida (expressa em escala logarítmica) mostrou-se linearmente relacionada $\left(R^{2}>0,82\right)$ com o tempo de incubação. Tal cinética de dissipação tem sido comumente verificada para pesticidas em solo, conforme observado por BARBOSA e RIGITANO (1994) para o aldicarbe, BROMILOW, et al. (1999) para fungicidas triazóis, SCORZA JÚNIOR et al. (2004) para o bentazon e o imidacloprid. De posse dos valores das constantes de dissipação do tiametoxam (k), calculados a partir das equações de regressão linear entre o logarítmo neperiano da quantidade do composto e o tempo de incubação $\left(\ln Q=-k t+\ln Q_{0}\right)$, foram calculados os valores de meia-vida do composto nos diferentes solos/tratamentos $\left(T_{50}=0,693 \mathrm{k}^{-1}\right)$ (Tabelas 1 e 2 ).

O teste de homogeneidade de equações de regressão não revelou diferenças nas curvas de dissipação do tiametoxam, quando comparados os dois níveis de umidade do solo $(p=0,9018)$. Por outro lado, a degradação do composto em solo esterilizado mostrou-se bem mais lenta do que em 
material não-esterilizado ( $p=0,0001)$. Tal fato evidencia a importância da atividade microbiana na taxa de dissipação do tiametoxam em solos.

\section{TABELA 1 - VALORES DE MEIA-VIDA DO INSETICIDA TIAMETOXAM EM AMOSTRAS DE LATOSSOLO VERMELHO AMARELO DISTROFÉRRICO (LVA $\left.{ }_{D F}\right)$ SUBMETIDAS A DIFERENTES TRATAMENTOS}

\begin{tabular}{cccc}
\hline Tratamento & Equação de regressão linear & $\mathbf{R}^{2}$ & Meia-vida (dias) \\
\hline $\begin{array}{c}\text { Solo esterilizado } \\
(19,4 \% \text { de umidade) }\end{array}$ & $y=-0,0027 x-1,5814$ & 0,82 & 257 \\
$\begin{array}{c}\text { Solo não-esterilizado } \\
(19,4 \% \text { de umidade) }\end{array}$ & $y=-0,0058 x-1,5654$ & 0,97 & 120 \\
$\begin{array}{c}\text { Solo não-esterilizado } \\
(30 \% \text { de umidade })\end{array}$ & $y=-0,0059 x-1,5700$ & 0,97 & 117 \\
\hline
\end{tabular}

${ }^{*} \mathrm{y}=$ In da quantidade remanescente de tiametoxam (mg).

${ }^{*} \mathrm{x}=$ tempo de incubação (dias).

Com relação aos efeitos da calagem e/ou fosfatagem, o teste de homogeneidade de equações de regressão revelou valor de p igual a 0,7303 para o efeito da calagem na dissipação do composto no $\mathrm{LV}_{\mathrm{d}}$. Assim, a calagem não afeta significativamente a degradação do tiametoxam nesse solo. Os resultados obtidos para o LVA, analisados estatisticamente para efeito da calagem e fosfatagem, revelaram valores de $p$ iguais a 0,0031 e 0,8493 para esses tratamentos, respectivamente, indicando que a calagem influenciou a degradação do tiametoxam nesse solo e que o efeito da fosfatagem não foi significativo.

De acordo MAIENFISCH et al. (2001), o tiametoxam é hidrolisado mais facilmente em solução aquosa com maior valor de $\mathrm{pH}$. Como os resultados mostraram valores de meia-vida maiores em condições de $\mathrm{pH}$ do solo mais elevado (Tabela 2) especula-se que a calagem possa ter influenciado a atividade microbiana em ambos os solos. Isso beneficiaria os microrganismos menos hábeis em degradar o tiametoxam, com efeito mais significativo no caso do LVA. Nas subamostras desse solo, submetidas à calagem e fosfatagem, verificou-se que a fosfatagem reduziu o efeito da calagem na dissipação do tiametoxam. Nesse tratamento pode ter ocorrido formação de fosfato de cálcio, que apresenta baixa solubilidade em água. Isso explicaria, ao menos em parte, o efeito antagônico da fosfatagem sobre aquele da calagem na dissipação do tiametoxam nesse solo.

Observou-se que o tempo de meia-vida do tiametoxam no LVA foi menor do que no LVA $\mathrm{e}$ $\mathrm{LV}_{\mathrm{d}}$, quando comparadas as amostras não submetidas à esterilização, calagem ou fosfatagem. Tal diferença pode estar relacionada com o fato dos solos $L V A_{d}$ e $L V_{d}$ terem sido utilizados alguns meses

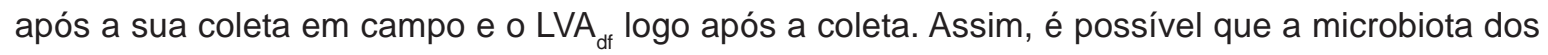
solos armazenados tenha sido afetada, diminuindo a taxa de dissipação do composto.

Os resultados obtidos mostraram alta estabilidade do inseticida tiametoxam em Latossolos brasileiros típicos. Os valores de meia-vida do tiametoxam nos solos estudados revelaram-se intermediários entre 47 e 385 dias, reportados por ANTUNES-KENYON \& KENNEDY (2001) para solos norte-americanos em condições de fotodegradação e na ausência de luz, respectivamente. No presente trabalho as amostras de solo foram incubadas com o tiametoxam sob condições de fotofase 
de 12h, simulando-se a aplicação do composto diluído em água na superfície do solo, conforme tem sido preconizado para a cultura do cafeeiro. Como tal condição é intermediária em relação às reportadas por aqueles autores, as taxas de dissipação do tiametoxam em Latossolos brasileiros típicos são comparáveis às verificadas em solos norte-americanos.

\section{TABELA 2 - VALORES DE MEIA-VIDA DO INSETICIDA TIAMETOXAM EM AMOSTRAS DE LATOSSOLO VERMELHO AMARELO DISTRÓFICO (LVA $)$ E DE LATOSSOLO VERMELHO DISTRÓFICO $\left(\right.$ LV $\left._{\mathrm{D}}\right)$ SUBMETIDAS A DIFERENTES TRATAMENTOS}

\begin{tabular}{ccccc}
\hline Solo/Tratamento & $\mathbf{p H}$ & Equação de regressão linear & $\mathbf{R}^{\mathbf{2}}$ & Meia-vida (dias) \\
\hline LVA $_{d}$ / COP0** & 4,1 & $y=-0,0033 x-1,5360$ & 0,95 & 210 \\
LVA $_{d}$ / COP1 & 4,6 & $y=-0,0032 x-1,5649$ & 0,84 & 217 \\
LVA $_{d}$ / C1P1 & 5,6 & $y=-0,0029 x-1,5248$ & 0,96 & 239 \\
LVA $_{d}$ / C1P0 & 5,4 & $y=-0,0023 x-1,5273$ & 0,97 & 301 \\
LV $_{d}$ / C0P0 & 4,6 & $y=-0,0025 x-1,5809$ & 0,88 & 277 \\
LV $_{d} /$ C1P0 & 5,4 & $y=-0,0023 x-1,5749$ & 0,88 & 301 \\
\hline
\end{tabular}

${ }^{*} \mathrm{y}=$ In da quantidade remanescente de tiametoxam $(\mathrm{mg})$.

${ }^{*} \mathrm{X}=$ tempo de incubação (dias).

${ }^{* *} \mathrm{CO}$ e PO indicam sem calagem e fosfatagem, respectivamente.

A alta estabilidade do tiametoxam nos solos estudados, associada à baixa adsorção do composto nesses solos (resultados ainda não publicados), indicam alto potencial de lixiviação desse inseticida nesses solos. Apesar do tiametoxam apresentar toxicidade relativamente baixa para mamíferos, a contaminação de recursos hídricos com resíduos desse inseticida pode afetar organismos aquáticos, principalmente insetos e outros artrópodes. Assim, estudos sobre a lixiviação do tiametoxam em solos brasileiros são necessários para que se possa melhor avaliar os riscos de impactos ambientais que o mesmo possa causar.

\section{CONCLUSÃO}

O tiametoxam mostrou-se bastante estável nos solos estudados, com valores de meia-vida entre 117 e 301 dias.

O efeito da calagem do solo sobre a dissipação do tiametoxam variou em função do tipo de solo. A variação no grau de umidade do solo, na faixa de 20 a 30\%, não exerceu efeito significativo sobre a dissipação do composto.

A atividade microbiana revelou-se como importante fator a determinar a taxa de dissipação do tiametoxam em solos.

\section{ABSTRACT}

\section{DISSIPATION OF THE INSECTICIDE THIAMETHOXAM IN SOILS FROM THE REGION OF LAVRAS-MG (BRAZIL)}

The objective of this work was to assess the dissipation of the insecticide thiamethoxam in samples of Latosols from the municipal district of Lavras, MG (Brazil), as well as the effects of liming, phosphate fertilization of the soil, microbial activity and soil humidity in this process. Subsamples of the soils were incubated with the insecticide at $25^{\circ} \mathrm{C}$, being periodically analysed for the remaining amount of thiamethoxam (by liquid chromatography). Dissipation of thiamethoxam in the studied soils followed first-order kinetics (half-life values from 117 to 301 days), showing a high stability of the compound. Liming of the soil reduced the dissipation rate of thiamethoxam in the case of dystrophic Red-Yellow Latosol, with the effect being 
partially reduced by phosphate fertilization. In the case of dystrophic Red Latosol, the effect of liming on dissipation of the insecticide was not significant. Dissipation rate was lower in sterilized soil material, showing that microbial activity is an important factor affecting degradation of thiamethoxam in soils.

KEY-WORDS: THIAMETHOXAM; DEGRADATION; LATOSOL.

\section{REFERÊNCIAS}

1 ANTUNES-KENYON, S.E.; KENNEDY, G. Thiamethoxam: a new active ingredient review. Boston: Massachusetts Pesticides Bureau, Department of Food Agriculture, 2001. 37 p.

2 AYRES, M.; AYRES JR., M.; AYRES, D.L.; SANTOS, A.S. Bio Estat 2.0: aplicações estatísticas nas áreas das ciências biológicas e médicas. Belém: Sociedade Civil Mamirauá; Brasília: CNPq, 2000. 272 p.

3 BARBOSA, T.M.L.; RIGITANO, R.L. de O. Influência da classe e profundidade do solo na degradação do inseticidanematicida aldicarbe. Pesquisa Agropecuária Brasileira, v.21, p. 955-960, 1994.

4 BROMILOW, R.H.; EVANS, A.A.; NICHOLLS, P.H. Factors affecting degradation rates of five triazole fungicides in two soil types: 1. Laboratory incubations. Pesticide Science, v. 55, p.1-6, 1999.

5 DÍAZ, R.D.; GAGGI, C.; SANCHES-HERNANDEZ, J.C.; BACCI, E. The role of soil and active ingredient properties in degradation of pesticides: a preliminary assessment. Chemosphere, v.30, n.12, p. 2375-2386, 1995.

6 DOMAGALSKY, J.L.; DUBROVSKY, N.M. Pesticide residues in ground water of the San Joaquin Valley, California. Journal of Hydrology, v.130, p.299-338, 1992.

7 EMBRAPA. Empresa Brasileira de Pesquisa Agropecuária. Centro Nacional de Pesquisa de Solos. Manual de métodos de análises de solo. Rio de Janeiro, 1997, 212 p.

8 LAVORENTI, A.; PRATA, F.; REGITANO, J.B. Comportamento de pesticidas em solos: fundamentos. In: CURI, N.; MARQUES, J.J.G. de S. e M.; GUILHERME, L.R.G.; LIMA, J.M. de; LOPES, A.S.; ALVAREZ, V.H. (eds.). Tópicos em ciência do solo. Viçosa: Sociedade Brasileira de Ciência do solo, 2003. v. 3. p.335-400.

9 LEISTRA, M.; BOESTEN, I.I.T.I. Pesticide contamination of groundwater in Western Europe. Agriculture, Ecossystem and Environment, v.26, p.369-389, 1989.

10 MAIENFISCH, P.; ANGST, M.; BRANDL, F.; FISCHER, W.; HOFER, D.; KAYSER, H.; KOBEL, W.; RINDLISBACHER, A.; SENN, R.; STEINEMANN, A.; WIDMER, H. Chemistry and biology of thiamethoxam: a second generation neonicotinoid. Pest Management Science, v.57, p.906-913, 2001.

11 NOVARTIS Crop Protection AG. Determination of CGA 293343 and CGA 322704 by HPLC: residue method REM 179.03. Basélia, 1998. 46 p.

12 NÓBREGA, J.C.A.; LIMA, J. M.; GUERREIRO, M. C.; RIGITANO, R.L.O.; MORENO, S.A.C. Retenção do fungicida triadimenol em latossolos em razão da calagem e da fosfatagem. Pesquisa Agropecuária Brasileira, Brasília, v. 40, n.5, p. 503-511, maio 2005.

13 RACKE, K.D.; SKIDMORE, M.; HAMILTON, D.; UNSWORTH, J.B.; MIYAMOT, J.; COHEN, S.Z. Pesticide fate in tropical soils (Technical Report). Pure and Applied Chemistry, v.69, p.1349-1371, 1997.

14 RIGITANO, R.L. de O.; GOUVÊA, A.V. Contaminação de manancial hídrico com resíduos de inseticidas em Maria da Fé - MG. In: CONGRESSO BRASILEIRO DE ENTOMOLOGIA, 15., Caxambu, 1995. Anais... Lavras: SEB/UFLA, 1995. p.485.

15 ROBINSON, P. Evaluation of the new active thiamethoxam in the product Cruiser $350 \mathrm{FS}$ insecticide seed treatment. Camberra: National Registration Authority for Agricultural and Veterinary Chemicals, 2001. 35 p.

16 SCORZA-JÚNIOR, R.P.; SMELT, J.H.; BOESTEN, I.I.T.I.; HENDRIKS, R.F.A.; VANDER ZEE, S.E.A.T.M. Vadose zone processes and chemical transport: preferencial flow of bromide, bentazon and imidacloprid in a dutch clay soil. Journal of Environmental Quality, v.33, p.1473-1485, 2004. 